\title{
Botany without bias
}

In the Gospel According to Matthew Chapter seven, Verse five, Jesus says "first cast out the beam out of thine own eye; and then shalt thou see clearly to cast out the mote out of thy brother's eye". We should remember this entreaty before too casually casting accusations of 'plant blindness'.

L

anguage usage helps maintain unconscious biases. In plant biology for example, there is the careless use of the term 'higher plants', without thinking about its meaning or implication. If there is a definition of 'higher plants' then it is synonymous with vascular plants, but the image it conjures is of upstanding, leafy land-dwelling plants. The problem is that 'higher' is a charged term implying superiority of this group over their non-vascular cousins.

This stratification is a manifestation of orthogenesis, the idea that evolution has both a direction and a goal. A perfect illustration of orthogenesis is the frequent meme of The Road to Homo Sapiens, the original version of which was drawn by Rudolph Zallinger for a 1965 edition of Life Nature Library ${ }^{1}$. Also known as The March of Progress, it shows a line of assumed human ancestors, starting with a gibbon-like Pliopithecus, processing from left to right, becoming taller and more upright in stance, and culminating in a modern human. The implication is clear, our evolutionary ancestors are only of interest as waypoints on a journey whose destination is us.

This is not how evolution works. Evolution diversifies and adapts species to better fit them to environmental conditions prevailing at a specific time; a chain of temporary kludges. No organism alive today can be said to be more or less primitive than any other as they have all been evolving for exactly the same 3.5-3.8 billion years since the time of LUCA, the last universal common ancestor. Viewed in this way, no plant can be claimed to be higher than any other.

Even taking 'higher plants' to mean 'vascular plants' is problematic. Mosses, for example, would generally fall outside the vascular category, and yet we published only this March a study showing that the widely spread Common Haircap Moss (Polytrichum commune) has a vascular system with many of the essential characteristics of xylem ${ }^{2}$. The clade of vascular plants may have grown to be the dominant form of land plants, but, as Jeffrey Duckett and Silvia Pressel write

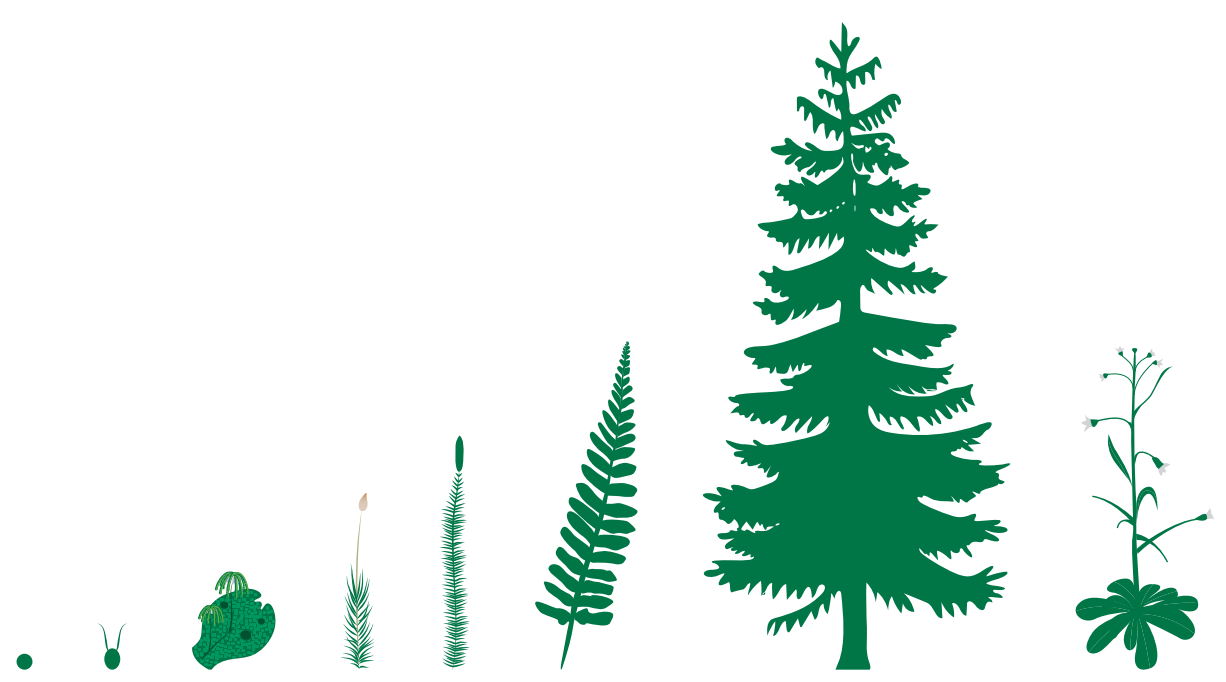

in their News and Views", "innovations associated with improving water use efficiency [...] may be more fundamental to the evolution of vascular plants than the vascular system from which they derive their name".

If plant biologists feel their object of fascination is overlooked and undervalued, spare a thought for the mycologists who rarely even have a department of their own to work in, being mostly lumped in with pathologists or microbiologists. As Merlin Sheldrake argues in his new book, Entangled Life: How Fungi Make our Worlds, Change our Minds and Shape our Futures (reviewed this month by Nick Talbot of the Sainsbury Lab in Norwich, UK), despite underpinning the nutrition of the majority of plants (and thus all land animals as well), plant biologists are frequently as 'fungus blind' as the population in general is " plant blind'.

At Nature Plants, we try to adopt a Catholic attitude to what constitutes a plant, and more importantly what will be of interest to plant biologists. In 2020 alone we have published genomic studies of hornworts ${ }^{4,5}$ and the streptophyte algae Mesostigma viride and Chlorokybus atmophyticus ${ }^{6}$. Even so, these studies spend much of their discussions on what the similarities of these organisms to angiosperms can tell about the history of plants' colonization of dry land, and much less on their characteristics and remarkable features. Even this month's study by Mitsuyasu Hasebe and colleagues, exploring how strand breaks in DNA stimulate the reprogramming of stem cells in Physcomitrella patens, is using the moss as a model for the process, its unique biology being an aid to methodology rather than the focus of the research.

This is not in any way to detract from the importance of those studies, but the fact remains that natural selection has no plan and plays no favourites. To understand the natural world that it has created we must keep our eyes open to all its diversity and strive to value every form of life within it.

Published online: 11 September 2020 https://doi.org/10.1038/s41477-020-00777-x

References

1. Howell, F. C. Early Man 41-45 (Time Life, 1965).

2. Brodribb, T. J. et al. Nat. Plants 6, 273-279 (2020)

3. Duckett, J. G. \& Pressel, S. Nat. Plants 6, 184-185 (2020).

4. Zhang, J. et al. Nat. Plants 6, 107-118 (2020).

5. Li, F. et al. Nat. Plants 6, 259-272 (2020).

6. Wang, S. et al. Nat. Plants 6, 95-106 (2020). 